\title{
Diagnostic relevance of autoantibody detection against inhibitors of apoptosis proteins in colon cancer and colon adenoma
}

\author{
YUKIE HOSONO, MAKI GOTO, DAISUKE KOBAYASHI, KAGEAKI KURIBAYASHI, \\ MAKI TANAKA and NAOKI WATANABE
}

Department of Clinical Laboratory Medicine, School of Medicine, Sapporo Medical University, Sapporo, Hokkaido 060-8543, Japan

Received September 2, 2014; Accepted December 5, 2014

DOI: $10.3892 / \mathrm{mco} .2015 .502$

\begin{abstract}
Autoantibodies against cancer-related antigens may be detected in the sera of patients with various types of cancer, although their clinical utility has not yet been established. In this study, we aimed to demonstrate the diagnostic relevance of autoantibody detection against inhibitor of apoptosis protein (IAP) family members in colon cancer, as compared to anti-p53 antibody, carcinoembryonic antigen (CEA) and carbohydrate antigen 19-9. We established an ELISA system using original recombinant proteins of IAP family members (survivin, livin and X-linked IAP) and measured the expression levels in the sera of 62 healthy donors, 250 patients with colon polyps (adenoma) and 176 patients with colon cancer. When the cutoff value was set as the mean value +2 standard deviations in healthy donors, anti-survivin exhibited the highest positivity rate (24.4\%) among IAP autoantibodies in cancer patients. Furthermore, the anti-survivin antibody exhibited a high positivity rate in early-stage carcinoma and adenoma. In the combination assay, reflecting the significantly high positivity rate of CEA in stage IV tumors, the positivity rate was highest when combining the detection of anti-survivin antibody and CEA in cancer patients (50.0\%), indicating that this combination may not be useful for the diagnosis of early-stage cancers. By contrast, reflecting the complete independence of anti-survivin and anti-p53 antibodies, the combination of detecting these two antibodies resulted in the highest positivity rate (35.6\%) in early-stage disease (stage $0-\mathrm{I}$ ). These results suggest that the combined
\end{abstract}

Correspondence to: Dr Naoki Watanabe, Department of Clinical Laboratory Medicine, School of Medicine, Sapporo Medical University, South-1, West-16, Chuoh-ku, Sapporo, Hokkaido 060-8543, Japan

E-mail: watanabn@sapmed.ac.jp

Abbreviations: IAP, inhibitor of apoptosis protein; CEA, carcinoembryonic antigen; CA19-9, carbohydrate antigen 19-9; XIAP, X-linked IAP

Key words: autoantibody, inhibitor of apoptosis protein, colon cancer, colon polyp measurement of anti-survivin and anti-p53 antibodies may be useful for the detection of early-stage colon cancer.

\section{Introduction}

Previous studies have demonstrated that inhibitors of apoptosis proteins (IAPs), such as survivin, livin and X-linked IAP (XIAP), are expressed in high concentrations in various cancers and hematological malignancies (1-4). In addition, survivin acts not only as an anti-apoptotic factor, but also plays an important role in cell proliferation and escape from the immune surveillance system via the induction of human telomerase reverse transcriptase and Fas ligand $(5,6)$. Other members of IAPs, particularly livin and XIAP, play distinct roles in various types of cancer $(7,8)$. Consequently, these facts suggest that detection of IAPs may be useful as tumor markers. However, tissue specimens or cells are required for the detection of IAP mRNA or proteins and, therefore, the clinical utility of this detection method is limited.

Several studies demonstrated the presence of autoantibodies against various cancer antigens in the peripheral blood. We also previously reported that autoantibodies against survivin and livin were detected in patients with gastrointestinal, lung, breast and hepatic cancers (9-13). Other studies also reported survivin autoantibody positivity in various cancers (14-18). However, there have been no comprehensive studies establishing an ideal detection combination regarding IAPs and other tumor markers. Furthermore, IAP positivity in patients with precancerous conditions remains unknown. Therefore, we targeted colon cancer and adenomas and investigated the positivity of autoantibodies against survivin, livin and XIAP, as well as the presence of anti-p53 antibodies $(19,20)$, carcinoembryonic antigen (CEA) and carbohydrate antigen 19-9 (CA19-9). In order to determine the ideal assay for the detection of colon cancer, we compared the positivity rates of several combinations of these markers.

\section{Materials and methods}

Patients. Blood samples were collected from 250 patients with untreated colon adenoma and 176 patients with untreated colon cancer at the Sapporo Medical University Hospital and related hospitals. Histological diagnosis was performed by expert 
doctors at a pathology center. As a control, blood samples were collected from 62 non-hospitalized adults without any malignancy. Informed consent was obtained from all the blood donors. Following blood centrifugation, the sera were divided into aliquots and stored at $-80^{\circ} \mathrm{C}$. The patients with colon cancer were diagnosed with stage I-IV tumors on the basis of the Union for International Cancer Control TNM classification guidelines (21).

Preparation of recombinant protein. mRNA was extracted from the SW480 colon cancer cell line (RNeasy Mini kit; Qiagen, Hilden, Germany) and cDNA was prepared (SuperScript First-Strand Synthesis System for RT-PCR; Invitrogen, Carlsbad, CA, USA) for gene amplification of full-length survivin, livin and XIAP. Subsequently, polymerase chain reaction (PCR) was performed with primers to amplify each gene. The primers for survivin (NM001168), livin (AF311388) and XIAP (U45880) were as follows: Survivin: forward, 5'-CACCATGGGTGCCCCGACGTTG-3' and reverse, 5'-TCAATCCATGGCAGCCAGCTGCT-3'; livin: forward, 5'-CACCATGGGACCTAAAGACAGTG-3' and reverse, 5'-CTAGGACAGGAAGGTGCGCAC-3'; and XIAP: forward, 5'-CACCATGACTTTTAACAGTTTTGA-3' and reverse, 5'-TTAAGACATAAAAATTTTTTGCTTGAA-3'. The PCR conditions were as follows: Taq polymerase activation at $94^{\circ} \mathrm{C}$ for $5 \mathrm{~min} ; 35$ cycles at $94^{\circ} \mathrm{C}$ for $15 \mathrm{sec}$, at $55^{\circ} \mathrm{C}$ for $45 \mathrm{sec}$ and at $72^{\circ} \mathrm{C}$ for $45 \mathrm{sec}$; and final extension at $72^{\circ} \mathrm{C}$ for $10 \mathrm{~min}$. Each band of amplified PCR product in the gel was extracted and then inserted into the protein expression vector pRT151 (Champion pET Directional TOPO Expression kits; Invitrogen). The inserted vector was transformed using BL21 competent cells. Each protein was purified using the HisTrap FF crude kit (GE Healthcare Bio-Sciences AB, Uppsala, Sweden).

Commercial antigens and antibodies. For the pre-absorption test and for drawing standard curves, the purchased antigens and antibodies were as follows: Recombinant human survivin (cat. no. 4160-50; BioVision, Inc., Milpitas, CA, USA), recombinant human livin (cat. no. 01-2103; American Research Products Inc, Grandville, MI, USA), recombinant human XIAP (cat. no. 822-XF-050; R\&D Systems, Minneapolis, MN, USA), rabbit-anti human survivin (R\&D), mouse anti-human livin (Abcam, Cambridge, MA, USA) and mouse anti-human XIAP (R\&D).

ELISA. Purified recombinant survivin (1 mg/l), livin (5 mg/l) and XIAP (1 mg/l) were used as antigens for coating wells in ELISA, which were placed in the wells of 96-well plates (Corning, Corning, NY, USA) and incubated overnight at $4^{\circ} \mathrm{C}$. After removing the antigen solutions, the plate was washed with Block Ace ${ }^{\mathrm{TM}}$ (Dainippon Sumitomo Pharma, Inc., Osaka, Japan) and the plates were blocked using Block Ace for $2 \mathrm{~h}$ at room temperature. After emptying the wells and washing 4 times with $0.5 \mathrm{ml} / 1$ Tween-20 [T-phosphate-buffered saline (PBS)], $100 \mu \mathrm{l}$ of diluted serum sample (1,000-fold) in 4-fold diluted Block Ace by PBS was added to each well and incubated for $1 \mathrm{~h}$ at room temperature. The samples were then removed and the wells were washed 4 times with T-PBS, after which of rabbit anti-human $\operatorname{IgG}$ (1:6,000 dilution) conjugated
$\mathbf{A}$

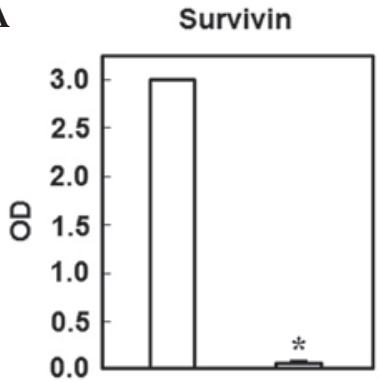

C

$\mathbf{B}$

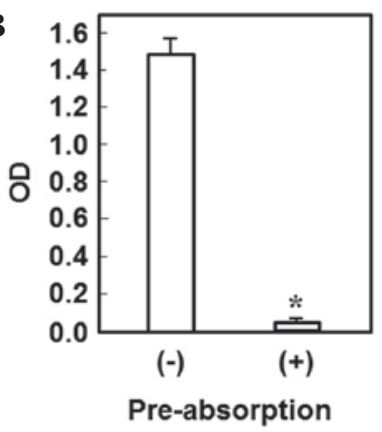
Livin

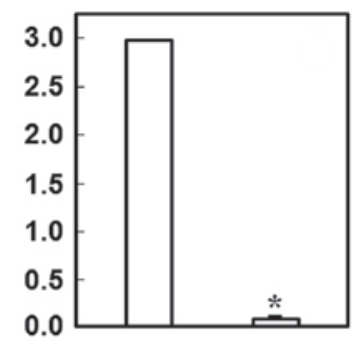

D

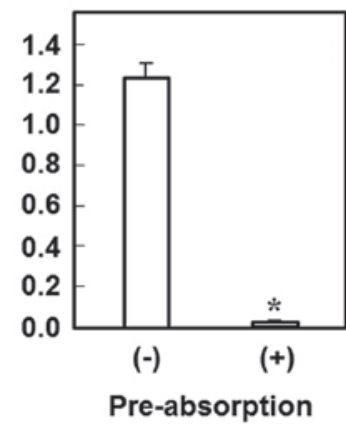

Figure 1. Pre-absorption test of antibodies with (A and B) recombinant survivin or $(C$ and $D)$ livin. (A and $C$ ) The antibody was pre-incubated with our recombinant survivin or livin and then allowed to react with commercially available recombinant survivin or livin. (B and D) The antibody was pre-incubated with commercially available recombinant survivin or livin and then allowed to react with our recombinant survivin or livin. ${ }^{*} \mathrm{P}<0.001 \mathrm{using}$ the Student's paired t-test. OD, optical density.

with horseradish peroxidase (Dako, Carpinteria, CA, USA) was added to each well and incubated for $30 \mathrm{~min}$ at room temperature. Following removal of this antibody solution and washing 4 times with T-PBS, each well was developed using tetramethylbenzidine (TMB). After a 30-min incubation in darkness, the reaction was stopped by adding $0.5 \mathrm{~mol} / 1 \mathrm{H}_{2} \mathrm{SO}_{4}$ and absorbance was measured at 450/620 nm using EVOLIS ${ }^{\mathrm{TM}}$ system (Bio-Rad, Hercules, CA, USA). The measured absorbance was converted to values using the standard curve. The measurement of the anti-p53 antibody was performed using the MESACUP ${ }^{\mathrm{TM}}$ anti-p53 test kit (Medical and Biological Laboratories, Co., Ltd., Nagoya, Japan) according to the manufacturer's instructions.

Measurement of concentrations of CEA and CA19-9. The serum concentrations of CEA and CA19-9 were measured by the reagent ECLusys ${ }^{\circledR}$ CEA (Roche Diagnostics Japan, Tokyo, Japan) and ECLusys CA19-9 (Roche Diagnostics Japan) on cobas $^{\circledR} 8000$ e series (Roche Diagnostics Japan).

Epitope analysis for survivin. The constructed survivin expression vector was used as a template and the expression vectors for exons 1-2, 3 and 4 of each protein were constructed using the following primers: Survivin 1: forward, 5'-CACCATGGGTGCCCCGACGTTG-3' and reverse, 5'-TCATATGGGGTCGTCATCTGG-3'; survivin 2: forward, 5'-CACCGAGGAACATAAAAAGCA-3' and reverse, 5'-TCAAATTTTGTTCTTGGCTCT-3'; and survivin 3: forward, 5'-CACCCTGGACAGAGAAAGAGCC-3' and reverse, 5'-TCAATCCATGGCAGCCAGCTGCT-3'. The recombinant protein for each exon was purified using the HisTrap FF crude kit (GE Healthcare Bio-Sciences AB) following transformation 
$\mathbf{A}$

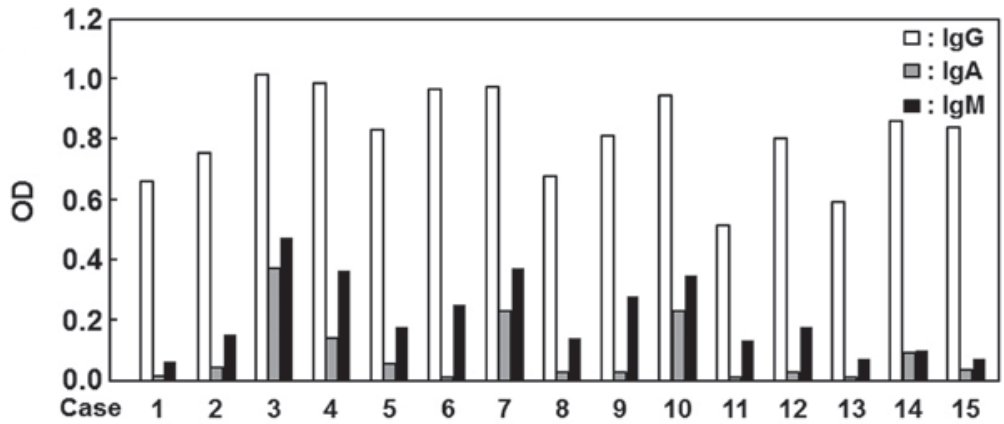

B

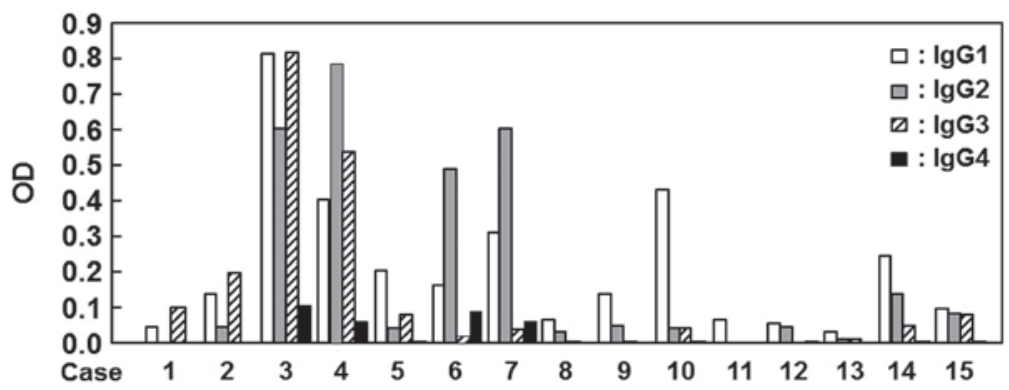

Figure 2. (A) Isotype analysis for anti-survivin antibody. Sera obtained from 15 patients with colon cancer were used for the analysis of immunoglobulin isotyping. For this analysis, the reactivities of $\operatorname{IgG}, \operatorname{IgA}$ and $\operatorname{IgM}$ were assessed among the patients. (B) Subclass analysis for anti-survivin antibody. Sera obtained from 15 patients with colon cancer were used for the analysis of IgG typing. For this analysis, the reactivities of IgG1, IgG2, IgG3 and IgG4 were assessed among the patients. OD, optical density.

by BL21 competent cells. ELISA was performed using these proteins and the reactivity of the sera obtained from the patients was assessed.

\section{Results}

Pre-absorption test. The commercially available antibody was pre-incubated with the commercially available recombinant antigen $(30 \mathrm{mg} / \mathrm{l})$ for $2 \mathrm{~h}$ at $37^{\circ} \mathrm{C}$ and then subjected to ELISA using the plate coated with our recombinant antigen, to determinine the analytical specificity of our own recombinant protein for each antigen. In addition, we also performed ELISA using the plate coated with the commercial recombinant antigen. For survivin and livin, both experiments revealed a significant decrease in the measured value, indicating absorption of the antibody (Fig. 1). However, for XIAP, the reactivity between the commercially available antibody and antigen was low and, therefore, the experiment could not be performed (data not shown).

Analysis of autoantibody isotype and subclass. Using sera from 15 patients with colon cancer, we analyzed the subclasses of the anti-survivin autoantibody that reacted to their own recombinant antigens. Among $\operatorname{IgG}, \operatorname{Ig} \mathrm{A}$ and $\operatorname{IgM}$, the difference in reactivity was the least for IgG (Fig. 2A). Moreover, the differences in the IgG subclasses were examined and IgG1 exhibited the least significant difference among all patients compared to IgG2, IgG3 and IgG4 (Fig. 2B).

Epitope analysis. Using the recombinant proteins for exons 1-2, 3 and 4 of survivin, the difference in reactivity was examined in 6 patients with colon cancer. As a result, apparent reactivity was observed in the sets for all exons and all patients, leading to a higher measured value compared to the negative control
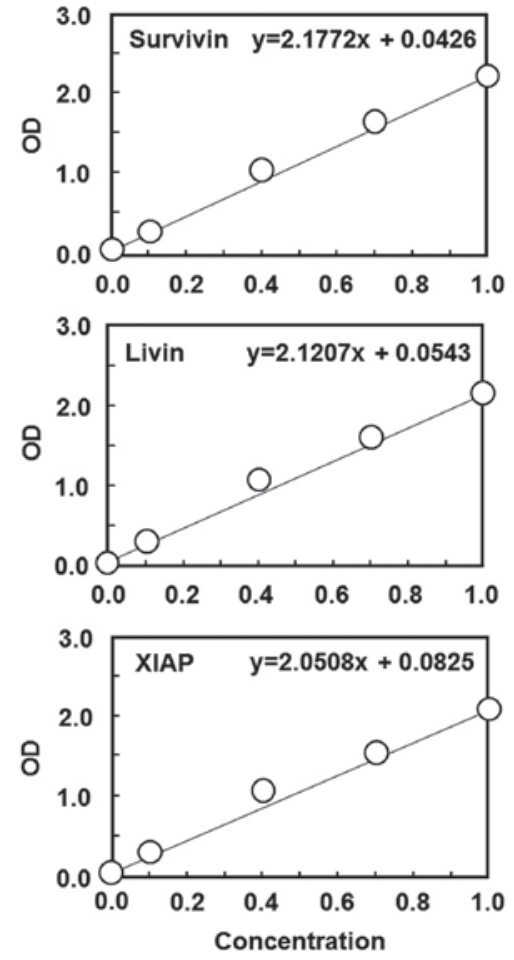

Figure 3. Standard plot for autoantibody detection against survivin, livin and $\mathrm{X}$-linked inhibitor of apoptosis protein (XIAP). The horizontal axis shows the antibody concentration. The highest concentration of each antibody was estimated as 1.0. OD, optical density.

samples without sera (data not shown). Consequently, no apparent specificity in the reactivity of antibodies in the sera was observed and it was suggested that our ELISA system for survivin has universality from the point of potential clinical use. 
Table I. Comparison of the positivity $(\%)$ in the single or combination assay at different clinical stages.

\begin{tabular}{|c|c|c|c|c|c|c|c|}
\hline Assays & $\begin{array}{c}\text { Total } \\
(\mathrm{n}=176)\end{array}$ & $\begin{array}{l}\text { Stage } 0 \\
(\mathrm{n}=25)\end{array}$ & $\begin{array}{l}\text { Stage I } \\
(\mathrm{n}=20)\end{array}$ & $\begin{array}{c}\text { Stage } 0+\mathrm{I} \\
(\mathrm{n}=45)\end{array}$ & $\begin{array}{c}\text { Stage II } \\
(\mathrm{n}=47)\end{array}$ & $\begin{array}{l}\text { Stage III } \\
(\mathrm{n}=51)\end{array}$ & $\begin{array}{c}\text { Stage IV } \\
(\mathrm{n}=33)\end{array}$ \\
\hline Survivin $^{\mathrm{a}}$ & 24.4 & 16.0 & 15.0 & 15.6 & 23.4 & 29.4 & 30.3 \\
\hline Livin $^{\mathrm{b}}$ & 9.7 & 4.0 & 5.0 & 4.4 & 14.9 & 9.8 & 9.1 \\
\hline $\mathrm{XIAP}^{\mathrm{c}}$ & 21.6 & 12.0 & 10.0 & 11.1 & 23.4 & 21.6 & 33.3 \\
\hline $\mathrm{p} 53^{\mathrm{d}}$ & 29.0 & 0.0 & 45.0 & 20.0 & 27.7 & 33.3 & 9.1 \\
\hline CEA & 35.2 & 4.0 & 5.0 & 4.4 & 28.8 & 33.3 & 81.8 \\
\hline CA19-9 & 19.9 & 0.0 & 15.0 & 6.7 & 6.7 & 15.7 & 51.5 \\
\hline \multicolumn{8}{|l|}{ Survivin } \\
\hline$+\mathrm{CEA}$ & 50.0 & 20.0 & 20.0 & 20.0 & 44.7 & 54.9 & 84.8 \\
\hline +CA19-9 & 42.6 & 16.0 & 30.0 & 22.2 & 27.7 & 37.3 & 66.7 \\
\hline$+p 53$ & 42.0 & 16.0 & 60.0 & 35.6 & 46.8 & 49.0 & 33.3 \\
\hline+ Livin & 30.1 & 20.0 & 20.0 & 20.0 & 29.8 & 35.3 & 36.4 \\
\hline$+\mathrm{XIAP}$ & 29.0 & 16.0 & 15.0 & 15.6 & 31.9 & 33.3 & 39.4 \\
\hline \multicolumn{8}{|l|}{ Livin } \\
\hline$+\mathrm{CEA}$ & 39.8 & 8.0 & 10.0 & 8.9 & 40.4 & 41.2 & 48.5 \\
\hline +CA19-9 & 26.1 & 4.0 & 20.0 & 11.1 & 21.3 & 25.5 & 57.6 \\
\hline$+\mathrm{p} 53$ & 30.7 & 4.0 & 45.0 & 22.2 & 38.3 & 41.2 & 18.2 \\
\hline+ XIAP & 23.9 & 16.0 & 15.0 & 15.6 & 27.7 & 29.4 & 39.4 \\
\hline \multicolumn{8}{|l|}{ XIAP } \\
\hline$+\mathrm{CEA}$ & 44.9 & 16.0 & 15.0 & 15.6 & 46.8 & 41.2 & 84.8 \\
\hline +CA19-9 & 33.0 & 12.0 & 25.0 & 17.8 & 29.8 & 25.5 & 57.6 \\
\hline$+p 53$ & 37.5 & 12.0 & 55.0 & 31.1 & 46.8 & 41.2 & 18.2 \\
\hline
\end{tabular}

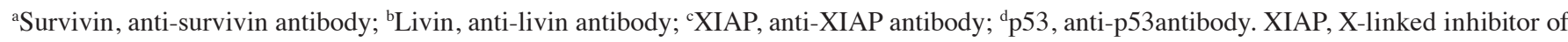
apoptosis protein; CEA, carcinoembryonic antigen; CA19-9, carbohydrate antigen 19-9.

Table II. Positivity (\%) in patients with colon adenoma $(n=250)$.

\begin{tabular}{lc}
\hline Assays & Positivity $(\%)$ \\
\hline Survivin $^{\mathrm{a}}$ & 18.4 \\
Livin $^{\mathrm{b}}$ & 2.4 \\
XIAP $^{\mathrm{c}}$ & 3.2 \\
p53 $^{\mathrm{d}}$ & 3.2 \\
CEA & 2.0 \\
CA19-9 & 1.2 \\
\hline
\end{tabular}

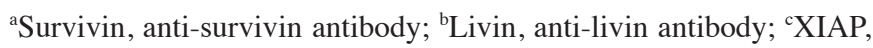
anti-XIAP antibody; ${ }^{\mathrm{d}}$ 553, anti-p53antibody. XIAP, X-linked inhibitor of apoptosis protein; CEA, carcinoembryonic antigen; CA19-9, carbohydrate antigen 19-9.

Standard curve. As seen in Fig. 3, our recombinant antigens and the diluted, commercially available antibodies for survivin, livin and XIAP were subjected to ELISA and used to draw a standard curve to obtain the actual values of serum antibody concentrations. We obtained an almost linear association for a wide range of concentrations for each set of antigen-antibody (Fig. 3).
Table III. Positivity (\%) of anti-IAP antibodies determined in each morphological type in patients with colon adenoma.

\begin{tabular}{lccc}
\hline Type $^{\mathrm{a}}$ & Total $(\mathrm{n}=250)$ & IAP $^{\mathrm{b}}(\mathrm{n}=56)$ & Survivin $^{\mathrm{c}}(\mathrm{n}=46)$ \\
Ip & $18(7.2)$ & $1(1.8)$ & $1(2.2)$ \\
Isp & $87(34.8)$ & $21(37.5)$ & $19(41.3)$ \\
Is & $91(36.4)$ & $26(46.4)$ & $20(43.5)$ \\
IIa & $48(19.2)$ & $7(12.5)$ & $5(10.8)$ \\
LST & $6(2.4)$ & $1(1.8)$ & $1(2.2)$ \\
\hline
\end{tabular}

Type: Ip, pedunculated; Isp, semi-pedunculated; Is, sessile; IIa, superficial elevated; LST, lateral spreading tumor. ' $\mathrm{b}$ IAP, positive for any anti-IAP antibodies. 'Survivin, positive for anti-survivin antibody. IAP, inhibitor of apoptosis protein.

Autoantibody expression profile in patient sera. The positivity of each antibody was assessed using a cutoff of the mean +2 standard deviations (SDs) of the measured values in healthy individuals to determine the clinical utility of autoantibodies against IAPs. In all the cancer patients, among all the IAP autoantibodies, the anti-survivin antibody exhibited the highest positivity rate using a single assay (Table I). The positivity rate of the survivin autoantibody was relatively lower 
compared to that of CEA, but was similar to that of the anti-p53 antibody. In the analysis by cancer stage, the positivity rate of the anti-survivin antibody was the highest in stage 0 (cancer in adenoma) of the tumor, but anti-p53 antibody was not detected in any of the patients. By contrast, the anti-p53 antibody exhibited the highest positivity rate in patients with stage I and CEA exhibited the highest positivity rate in patients with stage IV disease. We then compared the positivity using combinations of all the markers. In all the patients, the combination of anti-survivin antibody and CEA exhibited the highest positivity rate, leading to a diagnosis of $50 \%$ of cancers (Table I). Furthermore, in the analysis by stage, the combination of the anti-survivin antibody and CEA exhibited a significantly high positivity rate in stage IV tumors, reflecting the high positivity of only CEA. By contrast, for early-stage tumors (stages 0-I), there was complete independence between the anti-survivin and anti-p53 antibodies, resulting in the highest positivity rate (35.6\%) when testing the combination of the two. Furthermore, in 250 patients with colon adenoma, the anti-survivin antibody exhibited the highest positivity rate among all markers (Table II).

As regards the size of the adenoma in the patients positive for any anti-IAP antibodies, the average size $(6.4 \mathrm{~mm})$ was not significantly different compared to that in all the patients $(7.0 \mathrm{~mm})$. In addition, the average size $(6.5 \mathrm{~mm})$ in the patients positive for anti-survivin antibody did not differ compared to that in the patients positive for any anti-IAP antibodies (plotting data not shown). In the analysis of positivity determined by morphological type, there was no significant difference between the patients positive for any anti-IAP antibodies and those positive for anti-survivin antibody (Table III).

\section{Discussion}

In this study, we demonstrated the diagnostic relevance of detecting autoantibodies against IAPs, particularly survivin, as compared to other tumor markers in colon cancer. In the comparison among IAPs, the positivity in a single assay was the highest (24.2\%) for anti-survivin antibody. As regards the positivity of anti-survivin antibody, Rohayem et al (14) reported a relatively lower positivity rate $(8.2 \% ; 4 / 49)$ in patients with colorectal cancer using their ELISA protocol. By contrast, Chen et al (18) reported high positivity (sensitivity, 56.9\%) in patients with colon cancer. This significant difference by different investigators is mainly attributed to the criteria for establishing a cutoff value. Rohayem et al (14) established a strict cutoff of the mean +3 SDs and their data lead to a higher positivity when the cutoff was set as the mean +2 SDs. Chen et al (18) established the cutoff using different criteria (Yowden's index from receiver operating characteristic curve analysis: sensitivity, +; specificity, -1); therefore, healthy volunteers exhibited a higher positivity rate (percentage of pseudo-positive patients, 35.9\%) compared to the results of our study $(8.1 \% ; 5 / 62$; actual plotting data not shown).

As regards other IAPs, anti-XIAP antibody also exhibited a relatively high positivity rate; no significant increase in positivity was observed with the combination of anti-XIAP and anti-survivin antibodies $(29.0 \% ; 51 / 176)$, reflecting the similar expression profile of these antibodies (actual plotting data not shown). By contrast, the ability of anti-livin antibody to detect colon cancer was apparently low throughout all stages, which was consistent with the relatively lower expression of the livin protein in colon cancer (22).

As described in previous studies $(9,14,15,20)$, the anti-p53 antibody may also detect colon cancer and we observed a similar positivity for anti-p53 and anti-survivin antibodies. As regards the expression profile of anti-survivin and anti-p53 antibodies, the positivity in the combination assay in each stage of the tumor has not been elucidated. Our study demonstrated the complete independence in the reactivity of anti-survivin and anti-p53 antibodies, resulting in the highest positivity rate when tested together, particularly in early-stage colon cancer (stages 0-I). An advantage in measuring anti-survivin antibody was also shown for other stages, particularly stage IV, in which we confirmed a different positivity rate for the anti-survivin antibody compared to the anti-p53 antibody. These findings are considered to be consistent with the low correlation between the two antibodies that was reported in the study by Rohayem et al (14). On combining with other well-established tumor markers, CEA and anti-survivin antibody exhibited the highest positivity among IAPs, reflecting the significantly high positivity rate of CEA in advanced-stage tumors.

In the analysis using the patients with colon adenoma, anti-survivin antibody detected a higher number of patients compared to the anti-p53 antibody. Taken together with the results of patients with carcinoma, we established the significance of measuring anti-survivin antibody for the diagnosis of early-stage carcinogenesis. As regards application in the clinical setting, based on the results from patients with colon adenoma and cancer, the patients exhibiting positivity for anti-survivin antibody or for the combination of anti-survivin and anti-p53 antibodies should be investigated and treated by endoscopy.

In this study, we analyzed epitopes for their reaction with anti-survivin antibodies in patients' sera. Previous studies have identified several splicing variants resulting in protein deletion or additional protein insertion (23). When autoantibodies in the serum recognize only limited and specific epitopes, a limited number of patients with survivin variants may not be detected. Therefore, we constructed 3 recombinant proteins for exons 1-2, 3 and 4 of survivin and investigated the reactivity using patients' sera. In contrast to our predictions, sera from all 6 patients reacted to all recombinant proteins, indicating that the autoantibodies in the serum are able to recognize multiple epitopes, but not specific and limited epitopes. Consequently, we provided the first evidence on the non-specific reactivity of autoantibodies against survivin, indicating the universality of our ELISA system.

\section{Acknowledgements}

We would like to thank the medical staff of the Sapporo Kiyota Hospital and the Steel Memorial Muroran Hospital for the preparation of the patient's sera.

\section{References}

1. Gazzaniga P, Gradilone A, Giuliani L, Gandini O, Silvestri I, Nofroni I, Saccani G, Frati L and Aglianò AM: Expression and prognostic significance of LIVIN, SURVIVIN and other apoptosis-related genes in the progression of superficial bladder cancer. Ann Oncol 14: 85-90, 2003. 
2. Tanabe H, Yagihashi A, Tsuji N, Shijubo Y, Abe S and Watanabe N: Expression of survivin mRNA and livin mRNA in non-small-cell lung cancer. Lung Cancer 46: 299-304, 2004.

3. Takeuchi H, Kim J, Fujimoto A, Umetani N, Mori T, Bilchik A, Turner R, Tran A, Kuo C and Hoon DS: X-Linked inhibitor of apoptosis protein expression level in colorectal cancer is regulated by hepatocyte growth factor/C-met pathway via Akt signaling. Clin Cancer Res 11: 7621-7628, 2005.

4. Augello C, Caruso L, Maggioni M, et al: Inhibitors of apoptosis proteins (IAPs) expression and their prognostic significance in hepatocellular carcinoma. BMC Cancer 9: 125, 2009.

5. Asanuma K, Tsuji N, Endoh T, Yagihashi A and Watanabe N: Survivin enhances Fas ligand expression via up-regulation of specificity protein 1-mediated gene transcription in colon cancer cells. J Immunol 172: 3922-3929, 2004.

6. Endoh T, Tsuji N, Asanuma K, Yagihashi A and Watanabe N: Survivin enhances telomerase activity via up-regulation of specificity protein 1- and c-Myc-mediated human telomerase reverse transcriptase gene transcription. Exp Cell Res 305: 300-311, 2005.

7. Liu T, Brouha B and Grossman D: Rapid induction of mitochondrial events and caspase-independent apoptosis in survivin-targeted melanoma cells. Oncogene 23: 39-48, 2004.

8. Lopes RB, Gangeswaran R, McNeish IA, Wang Y and Lemoine NR: Expression of the IAP protein family is dysregulated in pancreatic cancer cells and is important for resistance to chemotherapy. Int J Cancer 120: 2344-2352, 2007.

9. Yagihashi A, Asanuma K, Nakamura M, Araya J, Mano Y, Torigoe T, Kobayashi D and Watanabe N: Detection of anti-survivin antibody in gastrointestinal cancer patients. Clin Chem 47: 1729-1731, 2001.

10. Yagihashi A, Asanuma K, Tsuji N, Torigoe T, Sato N, Hirata K and Watanabe N: Detection of anti-livin antibody in gastrointestinal cancer patients. Clin Chem 49: 1206-1208, 2003

11. Yagihashi A, Asanuma K, Kobayashi D, Tsuji N, Shijubo Y, Abe S, Hirohashi Y, Torigoe T, Sato N and Watanabe N: Detection of autoantibodies to livin and survivin in sera from lung cancer patients. Lung Cancer 48: 217-221, 2005.

12. Yagihashi A, Ohmura T, Asanuma K, Kobayashi D, Tsuji N, Torigoe T, Sato N, Hirata K and Watanabe N: Detection of autoantibodies to survivin and livin in sera from patients with breast cancer. Clin Chim Acta 362: 125-130, 2005.
13. Yagihashi A, Asanuma K, Kobayashi D, Tsuji N, Torigoe T, Sato $\mathrm{N}$ and Watanabe N: Autoantibodies to survivin in patients with chronic hepatitis and hepatocellular carcinoma. Autoimmunity 38: 445-448, 2005.

14. Rohayem J, Diestelkoetter P, Weigle B, Oehmichen A, Schmitz M, Mehlhorn J, Conrad K and Rieber EP: Antibody response to the tumor-associated inhibitor of apoptosis protein survivin in cancer patients. Cancer Res 60: 1815-1817, 2000.

15. Koziol JA, Zhang JY, Casiano CA, Peng XX, Shi FD, Feng AC, Chan EK and Tan EM: Recursive partitioning as an approach to selection of immune markers for tumor diagnosis. Clin Cancer Res 9: 5120-5126, 2003.

16. Karanikas V, Khalil S, Kerenidi T, Gourgoulianis KI and Germenis AE: Anti-survivin antibody responses in lung cancer. Cancer Lett 282: 159-166, 2009.

17. Uemura N, Kodama S, Nomi N, Okamoto T and Suzuki M: Correlation between anti-survivin antibody and survivin mRNA expression in head and neck cancer patients. Acta Otolaryngol 130: 959-965, 2010.

18. Chen JS, Chen KT, Fan WC, Yu JS, Chang YS and Chan EC: Combined analysis of survivin autoantibody and carcinoembryonic antigen biomarkers for improved detection of colorectal cancer. Clin Chem Lab Med 48: 719-725, 2010.

19. Lubin R, Schlichtholz B, Teillaud JL, Garay E, Bussel A and Wild CP: p53 antibodies in patients with various types of cancer: assay, identification, and characterization. Clin Cancer Res 1: 1463-1469, 1995.

20. Hammel P, Boissier B, Chaumette MT, Piedbois P, Rotman N, Kouyoumdjian JC, Lubin R, Delchier JC and Soussi T: Detection and monitoring of serum p53 antibodies in patients with colorectal cancer. Gut 40: 356-361, 1997.

21. Sobin LH, Gospodarowicz MK and Wittekind C (eds): TNM Classification of Malignant Tumours. 7th Edition. Wiley-Blackwell, pp100-109, 2009.

22. Ding ZY, Zhang H, Adell G, Olsson B and Sun XF: Livin expression is an independent factor in rectal cancer patients with or without preoperative radiotherapy. Radiat Oncol 8: 281, 2013.

23. Taubert H, Kappler M, Bache M, Bartel F, Kohler T, Lautenschlager C, Blumke K, Wurl P, Schmidt H, Meye A and Hauptmann S: Elevated expression of survivin-splice variants predicts a poor outcome for soft-tissue sarcomas patients. Oncogene 24: 5258-5261, 2005. 